\title{
Lapurdum
}

LAPURDUM Euskal ikerketen aldizkaria | Revue d'études basques |

Revista de estudios vascos | Basque studies review

$9 \mid 2004$

Numéro IX

\section{Jusef Egiategi erreformista (1785)}

\section{Txomin Peillen}

\section{OpenEdition}

\section{Journals}

\section{Édition électronique}

URL : http://journals.openedition.org/lapurdum/858

DOI : 10.4000/lapurdum. 858

ISSN : 1965-0655

\section{Éditeur}

IKER

\section{Édition imprimée}

Date de publication : 1 novembre 2004

Pagination : 215-226

ISBN : 2-68781-376-X

ISSN : $1273-3830$

\section{Référence électronique}

Txomin Peillen, « Jusef Egiategi erreformista (1785) », Lapurdum [Linean], 9 | 2004, Sarean emana----an 01 mars 2009, kontsultatu 20 avril 2019. URL : http://journals.openedition.org/lapurdum/858 ; DOI :

10.4000/lapurdum.858 


\title{
Jusef Egiategi erreformista (1785)
}

\author{
Txomin PEILLEN \\ UPPA - IKER-UMR 5478 du CNRS
}

Zuberotar filosofo apal horren obraz historia laburra egin behar dut. Pariseko Bibliothèque Nationaleko esku idazkien saltsa lehenik aipatu. Idazle horren lana frantses titulu baten pean aurkitu nuen : Le philosophe basque. Benetako izena Uskaldun filosofoaren ekheia da. Larramendizatu XVIII. mendeko zubereraz idatzia da. Berehalaxe konturatu nintzen hizkuntza traketsa ahazturik ideia jakingarriak zeuzkala. Lehen Libürü hori Euskaltzaindiak argitaratu zuen.

Handik laster kontsultatu nuen beste bilduma bitxia Les proverbes d'Oyhenart, copie du XVIIIe siècle eta liburu lodi bat ekarri zidaten, Oihenarten atsotitzek leku tcxikia dute idazkian, baina denbora berean barnean bi hizkuntzetan Egiategik bere klasetarako adelatu esaldi, errefrau eta iruzkinak bazeuden eta izenburua Mosde Etcheberriren gogamena. Lan horren hasiera berritan kopiatua testuan nahasian emana da, ez nuen zuzentzeko kemenik izan.

Geroago Patri Urkizu kantu biltzen zebilela Pariseko B.N. ospetsu horretan zegoen Chants guipuzcoans delako liburua eskatu zuen eta Egiategiren lehen liburuaren jarraipena edo Filosofo II bat aurkitu zuen kantuekin bildua.

Azken lan hau, zirriborroa da. Ahal bezala lau urteren buruan irakurri dut eta leheneko transkripzioa egin, oraiko grafian ipini eta fotokopiak Euskaltzaindiari musutrukean eman. Han lotan daude. Requiescant in Pace. Alta bigarren liburu hori da Egiategiren jakingarriena. Bi kapitulu aurkeztuko dizkitzuet, Egiategi nahiz apala izan, bere garaiko gizona izan zela erakusteko, baita erreformista eta ez iraultzaile.

Erreformista izenpean sailkatu dut zuberotar idazle hori eta hala ezarri badut, euskal gizarteaz eduki zuen ikuspegi zorrotza eta moralkeria gehiegi gabe eskaintzen dituen aterabideak erakusten dituelako.

Idazle horrek bere lanak Iraultza frantsesa baino lehen idatzi zituen. Garai hartako askoren gisa iturrien aipamenak ez ditu zehazten eta zuzenean euskaraz emaiten : humanitaten artean bizi da ; bestalde bizitu zituen zertzelada eta gorabeherekin lotzen ditu bere gogamenak.

Jakina Egiategik bere apaltasuna eta atrebentzia aitortzen ditu : Ha u eztianak irakurtuko et za io batere dolutuko baina orduan, frantsesez ere ez zen ipar Euskal herrian filosofiaz idazten, gutiago euskaraz. Ez da Voltaire edo Rousseauren mailara iristen : garai hartako euskal gizartearen mailan dago, halere mende hartako urduritasuna, arrenkuratasunaren berri ematen digu. Ez da hurrik ere iraultzalaria eta jazarpena ez du onartzen etxeko hega tza ri sü emaitea delako.

Bere euskalduntasuna agertzen da norberekeria, indibidualismoa modan delarik bera gaur komunitarismo izenez zentralistek deizten duten etnia elkartasun eta nortasuna goraipatzen baitu. 
Bere kritika gogorrena jadanik beste leku batean aipatu dudana da kontsumerismoak eta diruak jendeak usteltzen hasia dela.

Nola nahi orduko Argien mendeak edo ilustrazioak Egiategiren gogamenean eragin handia eduki dute. Euskaltzaindiko jardunaldietan, iaz, Atarratzen Intxausperekin erkatu nuen erakusteko XIX. mendeko Intxauspek izpiritu gibeloi ta kontserbatzailez Egiategik dionaren aurkakoa erraten duela.

Anitzetan Iparraldeko egoeraren berri ematen badigu ere nire harxedeak batikbat Egiategik zer dion gerlaz, armadak kontrolatu beharra, gudariez eta erlisio jasangarritasunaz ikertuko ditut.

\section{Gerlaz}

Ainitz irakurriz eta berak diona kontutan harturik, ondorioz atera dut, Egiategi Prusian eta Polonian soldado egon zela, hain zuzen bere liburua argitaratu behar zion Belako zaldunarekin. Beraz, Gerlaz, Bakiaz, Soldado edo gudarietzaz diona aztertuko dugu baina, zuzenean irakurgarri izan dadin, larramendismoak eta zuberotarrismoak gaurko euskaran jarriko ditut eta terminatxoak gakoen artean utziko.

Gerlaz kapitulua horrela hasten da.

Gerla da Jinkoak egorri dian (mikalta) izurrite latzena. Bethi izan ta izanen dena pholborraren sorlakiak diratekieno: khanbio handia dü jarri gudetan; noizpait harma thipilak eskian gudariak ziradian bata berziari hüillantzen (hurbiltzen) eta han bihoztoiari erramüa zegoan. Orai kanon ta ezkopeta'khaldiz ürrhünetik dirade harmadak gudatzen, hala gütiago jende da hiltzen ta bitoria merkiago dena erosten tieso a go bere lekian dago.

Ez zuen iragarri nola polborak armak eskuan baino hil anitzez gehiago eginen zuela. Egiategik Callot grabatzaileak edo Goyak eman gerla irudiak hitzez kontatzen dizkigu eta orain Afrikako herri askotan gertatzen dena oroitarazten :

Izan da nazio (dierri) batzu gerlan baizik etzirenak laket, hetarik Alemanak ta Thrazak, hartakoz Euripiodak deitzen dü Trazia Marwen herri. Erresuma bada gudariek harmak dütieno bethi garbi eta xahü, bena nontik jinen ereien gerla, ez bethi beha ta den gutien (demendren) astaküria n a taba laz edo tronpeta $z$ a ri direno gerlak berekin dütian lokarriak eztakie, herri osoki errer eztie dolürik, jendia oihanetan ihesi dütie ikhusten ta haurrak gosez hilik.

Hiritarrak ohitürik, binatze (mahasti) erreetzaz, etxalte (galkerredaz) honda tuez eztie axolik heek dirade gerlaren frütiak; alabadere gerla da sü bat behin phiztü denian ta lekhü bat erre berzia la la ister doana ta nekez hiltzen dena.

Egiategik, filosofo ainitzek bezala gogoko ditu irudiak, alegoriak, sinboloak eta hona batzuk:

Negüari üdüri Ekhia gureganik abiatzen deno, odeiez dü zelia belzten, airia izotzez loditzen, lürra (kharroaz) hormaz (herkhatzen) lehertzen, ostoak zühaneta rik erorten ta lürra bere edertarzünaz da (pikarraitürik) bilusik, orozbat ari da gerla haren (eretzian) nibelean : zian aberastarzüinetik herexarik eztereio üzten ta harez basa eremüa dü egiten, bere la ngiletzaz ba getürik dütialakoz hobenak (erho) hil. Milion bat gizon khosta zen Cosaren fama; zer fama errabiatia! Zonbat urthez halako galtzepena küperatü 
eztiren hiriak hutsik, legiak hilik, errelijionean (mengoan) ahuldua, (khaparrek) sasiek elgeak estaltzen ta dolamen beltzik baizik orotarik ez entzüten.

Egiategik badirudi erregek egiten dituzten gerrateak onartzen dituela eta lege, arauekin militarren arteko gauza bezala.

Gaistokeriak dialakoz gerla behartü bere legiak dütü. Ha la rik ihor baztert ezta itekina heetarik; ezpalitz, hala photeria lükianak gerla egin liro bere nahiala ta guda lizateke mündüan bidezaleak bezala oihanetan ohoinen artean, hartakoz ezta gerla lealik, printzen artian dena baizik. Heek beren arrazoak behar dütie ta harmak hartü beno lehen (ogenak) erruak izanik ere, ba ta ri arpezkiroz, ma nifesto deitzen direnetzaz, na hiz mündüa bere althe jarri, die beren züzenak edertzen ahalena, kanoan ezkiribüz dagoelarik ultima ratio regum hartan erregeren(tzat) azken arrazoa dagoela.

Gerlaren estakuru edo aitzakiak aztertzen ditu gero :

Bakiaz (akhi) nekatu diren printzek gerlaren astaküriak franko dütie : berrehün urthez hiri batez gozatzen delakoz, aüzoak ordüan arrapatü zeienak edo aüzoa sobera handitürik itzal egiten dianak edo adiskideak eskerniatzen badü printze (bakaltarri) despota batek. Zonbat aldiz errelijionearen ga tik dia la rik ha rek bakia pheredika tzen edo ardüra jeloskeriaz aüzoa delakoz sobera aberasten.

Egiategik Frantziaz eta gerlaz diona orain ere Estadu Batukoei eta frantses Errepublikari aplika daiteke, orduko frantses belizismoaz diolarik :

Bada (dierri) erresuma denboratik denborala gerlaz beharra dienik, frantzesa da heeta rik lehena. Aspaldian da errana ha mar urthekal odol ga istoa ren idokitzeko ezpada guda eraziten dela (hirotzen) usteltzen; gerla dü maite, non na hi den ha rat doa frantzesa laister, hartakoz ere nozpaitekoak zioan nullum bellum sine gallo, gerlarik frantzes bagerik etzela, net egia dena; ez berze batek beno herioaz eztian axolik, bena bai gauza (sic) berriak maite dütia la kotz.

Frantsesa ez da gerlari ona, egonezina eta pazientzia gabekoa delako :

Lan lüzea da frantzesaren (ozartezaren) pazentziaren eta (ezegokiaren) egonezinaren heztea, haren bulzadaz baliatzea (ekinarte) eraso handian ezi khea bezala (a uher) alfer dagoenean badoa, hartakoz da errana: güdaren hatsarrean gizon beno haboro dela ta azkentzean emazte beno gütiago ez lotsaren gatik bena haren (frantsesaren) bizita rzünak obra lüziak dütia lakoz hügüin ta bertan ez lotsa rengatik beita minkaizten. Beraren (ozartitzak) inpazientziak dü etsaiaren komediak beno akhitzen(ago)

Erran dianak shobeki akopilatzean (erasoan) ezi ez gordotzian (defentsan) ta Espa inola gordotzean akopilatzean beno"bi (dierriak) erresumak zütian ezagützen.

Hantik proba balore hotza beroa beno gerlan tiesoagoa dela. Cincus Sulpicius prokontsulak frantzesen (sic) kontra a ri zenian zioan etzia la etsa ia ren bere ezinegonaz hurtzen zianarekin deusere bentüratüren, zialakoz denbora faltsiak frantzes bulkada furorem gallicum deitzen ziana, hiltzen.

Gerla egiteko gudariak dirua behar du :

Bena zoin nahi dierrian (erresuman) zonbat nahi gudari den, diharü falta denean flaküki doatza obrak, diharüa delakoz gerlaren zaina ta ezi, kidari zühürrak zioan, gerlan hirur gauza behar ziradiala, ürrhe, zilhar eta kopre, hiruetarik diru egiten dena ta haren (mengoa) nahi bizia, dabila jarraikiz, hartakoz Augusta enberadore zühürrenak Senatari zian gogatü, bakean gerlan bezala sekülakoz harmaden 
betira undia rena egin ladin, thesoro ba tosorik egonen zena, ezi den gudaria nahi bezain bihoztoi behar güziak ezpadütü kobart da teke ja rri, bihoztoia go ere gertzen deusere falta eztianean ta kidariak zütienean zaitirantzen (?)

Gerla odoltsuen aurka agertzen da Egiategi bakezale armatua.

Lazedemonek etsaiak zutienean jokotrüa zonbaitez garhaitü Mars Jinkoari zezen bat zien oberta emaiten. Gudarien indarraz aldiz oilar bat baizik etzien eskentzen, hantik zielarik erakusten garaipen ederrenak ziradiala odol gütik ixuri zütianak, halakoxe Cosaren errana:

\section{Bakea nahi badïk (dio a dagioak) gerla a dela ezak}

Gudariak ezpadü dirurik, hartzen du, ofiziozko armada ongi ordaindua behar da :

Gerla aidürü dagoenari ardüra falta dereio egiten (..) hartakotz gudariak ahalik haboroenak ta aitzinetik diha rü behar zereiena, ezi zerbaiti falta küpera ezta ite Non licet in bello pecare.

Zesarrek entrenamenduaren balioa zekusan :

Cosar izan da khidari ta gudari mündüko handiena, berak zütian soldadoak ekireiten (?) bakean gerlan bezala. Ka sü güzietan zer egingei zian etzian bakhoitzik, noiz baraitx, noiz laster, noiz aitzina, noiz gibelerat, noiz lantzaz, noiz ezpataz, noiz algarreki(n) noiz berhez güda tü behar zien batere etzen etzakianik. Bera ere beharrean zen gudari ta khidari gerla handirik egin bazian ta loria hanitz bildü, erran daite(ke) etrziala bere bizian soldadorik manha tü berak beno lan ariman, gogoan ta khorpitzean gehiago ükhen zianik.

Soldado edo gudarietzaz

Egiategik lehen liburu hori idaztean parropiako errejenta dela dio, geroago bere burua kolejioko errejent bezala emaiten du eta gerla ikusi düala salatzen du. Hain zuzen armadarik beharbada ez da zernahi ofizial edo khidari behar, berriki Irakeko gertaerek oroitarazi digute. Hona zer dion Egiategik :

Diradealarik errejentak, soldadoen edo gudarien bürüzagi lehenenak, heetarik (khidarigeier) ofizialgaiei agintze zonbait dügün jar heben, heen ofizioan gizon handienez baliatü direnetarik, hartan batzütan ere gerthaldi ikhusi düdanetarik; ezi errejent baniz ere zerbait berze ofizioetan ikhusirik nago.

Egiategik dio soldadoak eta apezgaiak goi karguetara iristeko eskolatu beharra daukatela.

Soldadogoa gerlan, thonsüratüak diradianak Elizan, soldado ta a pezgei gazteak ka rgü handietara heltzeko düe bide lüzea ta gaitza, bena esperantzak garhaitzen düana ta jakiteak llabürtzen : ezta berze biderik haren beharra eztüanik; banuzke gerlak jakintze güziak nahi dütiana, ezi ezta (aldekairik) gaitasunik mündüan harmadan khidariak (bath) batu eztirokienik ta medika mementoan behar eztianik

Baina zer ikasi behar luke gudari buruak?

Han kasü güzietara dagoelarik aidürü, gudarien (a delatzea) formatzea dü errekeitü meheena, gerlaren ta bakearen legeetan jakintsü, zer diroan eta eztiroan egin, heek ezpadazki, deus egin ez liro; gerla zaharrak ezpadütü irakhurtü nola berria egin liroke, delakoz bethi ofizioa, berari üdüri. Hola gerlatik kanpo ogenik ez egitea, jendeen züzenetan ezpada jakintsü ta zer diradean obrak gerlak zilegitzen eztütianak. 
Soldado eta soldado burugaiaren kalitateak : obeditzen ikastea.

Gerlako lehen baldintza khidari hona eztaitekeala gerlan soldado ona izan eztena ta honki manha ez lirokeala, honki menpetï izan eztena. Bakhanki dirade gudariak bestazale delakoz gerlaren ofizioa la ngilena, haboroena astokin, hezkaitz, arrabuhin ta suspita kor. Bena errege ja unak, a pezak errelijionearen mantena tzeko ba dirade, eginbide hartan ere khidariek düe esküa etxekiten gudarieta n a rima ezin ederragorik ere, fedeari tiesorik bada.

Soldadoak goxokiegi bizi direnean :

Zilharkeriak dirade gerlan gauza (a uherrenak) alferrenak, heen begiratzeak errekeitü gehiago dü emaiten eziez gozamenak somoia(?) ekharten.

Carles XII Suediako erregeak ontzi la torrietan baizik etzüan jaten ez eta erre edaten arrazoi harengatik. Ardoak eta aigardentak sekülan eztüe gerlan senthagaillarik egin, dielakoz gizonaren bürüa nahasten ta indarrak trenkatzen. Egiazko balorea dagoelako gizonaren zentzü honean ta hotzean ta ez mememntoak düan borraskan : hartakoz ere Pesenniusek Egyptan Nilaren bazterretan ardoa züan debekatü ta koziner güziak akasatü.

Egiategik halere erran du gerlan behar diren gauza nagusien artean urrea dela, baina geroago dio urrea gudatu gabe herri batez edo hiri batez jabetzeko erabil daitekela.

Egiategik adibideak (gertaldiak) gogoko ditu, gero Alexandro Severo Spurius Albinus adibide on eman ondoren, ongi jatean suntsitu ziren Alexandro Handiaren, Annibalen Carles VIII Frantziakoen gertaldi okerrak ematen dizkigu Suediako erregeak nola Narvan Polonian gudukaturik Saxako herri ederrean :

Herri gizen hartan bere harmada erthailtürik soldadoak gizentürik, diharüz kargatürik Erruso enberadoreak mendikatü nahiz 1709. ko negü harrigarrian gosez eta hotzez jendeak ta kabaleak hiltzen zeitzeno Pultavara drano züan ja rraiki (...)

Egiategiren denboraz geroztik Suediako erregeari Josef Bonaparteri gertatu zitzaion, lapurreta gehiegiz kargaturik eta Espainian gizenturik Gasteizaldean frantsesek galdu zuten, la ba taille de Vitoria.

Urrunago Egiategik Coriolan aipatzen du :

Harmada errumana galkidatürik zen osoki Coriolan heltü zereionean khidari. (Dremenden) den gutien falta leger kastigo latzenetzaz zütialarik azotatzen. Soldadoa la nez züan phorrokatzen, bera burhas ta za ia a rhinean elhürraren ta kharroaren erdian zabilan ta bere apairüetan ez bertze hazkürririk soldadoena baizik, ahala Züan harmada hezitü ta manüetara menpetü.

Alderantziz gogorki kritikatzen ditu karroza ederretan, jantzi eda jan-edari ederrenetan bizi ziren garaiko frantses "khidariak".

Egiategirentzat soldadoen hirurgarren kalitatea ez beldurti izatea liteke

Güdarien zorthea da herioaren mespretzüan bizitzea; gauza aphürrenek alabadere lotsatzen diena ta txipikeria batez segürtzen dena, ez. Gure (dierria) erresuma da arhin ta khanbiakor, hartakoz mündü güzian Frantziari bezain bere banderetarik ihesi dabiltzanak galbide haren eginna hia da khidarietan dütiela kotz soldadoak (a uherkerian) alferkerian üzten ta delakoz gizon (a uherra) alferra debeiatzen lekhüz khanbioan laket. 
Gero Egiategik kondatzen du erromatar soldadoa nola lanean hezia zen eta Frantzian gertatzen dena hitz politekin iruzkintzen :

Mosde Louvois-en denboran la uzpabost harma ziradean Frantzian ta batetik berzeala "la isterkariak" hain üsü non errejimentüak elhürra bezala ekhian, beitziraden hurtzen"

Iheslariari kobarte izendatzeak geldiaraz lezake : hala dio Egiategik nola Annibalek berriz bildu zituen ihesi joaiten ziren hiru mila espainol :

Soldado gehiegi eta inperioak :

Egiategik dio aberastasunen biltzeko gerla egin zutela espainolek :

Ürrhe ta zilhar mendiak bezain gora herri heetarik düe a thera tü ta harrigarri gizon heetan ehortzi. Aberastarzün heek dirade abartü erresuma abegiazko dena dena dago oküpa türik ta lürrak langile bagerik".

Adibidetzat ematen du nola erromatar armadetan 17 urteekin sarturik hala nola Pompeak eta Cœsarek soldadoak gogorki erabiltzen eta :

50 urthegerrenean beren etxeta rat zütien igorraiten

Soldadoaren esperientzia eta adina.

Frantzian soldado denbora laburrez (zortzi urte) ez dute beren ofizioa ikasten, nahiz Solda do zaharrak berriak beno hobe direla ezta düdarik, arrazoaz bürüza gia a prendiza beno dela langileago, manü gaberik obra dakielakoz ta hartan tiesoago. Beraz ordüko Alemanak frantsesak beno hobe...

Soldadoak lanean bai, baina sarraskietan ez. Egiategik kontatzen du nola Alexandro Severo soldado eri ta zaurituez arduratzen zen esanaz :

hiri galdüak arrahartzen ziradea la ,bena ez gizon hilak arrapizten"

Armadak, inperioak, koloniagaiak

Egiategik uste baitzuen nobleek eta soldadogaiek liburua irakurriko zutela jarraitzen du idatziz lehen linean eztela, aitzinean, ez gudari zaharregirik ez eta gaztegirik jarri behar eta amaitzen du ikusi zituen Europako soldadoen uniformeak aipatuz eta Europako armaden indarrak iruzkinduz :

Oraiko egünean phodenta ta heek (Espainia, Suedia, Danemarka, Angleterra, Errusia) heek güziek düe milion bat zortzitan ehün mila laurhogei ta mila gudari, egünkal phaküa düenek ta hoinez edo zaldiz gerlarako adela türik daudenak. Europa mündüko la ur phartetarik bata bere printzetan photere düe, berze hirurak ga rha it liroana.

Bakeaz

Irudi goxoekin Egiategi soldado ohiak bakearen goraipamenarekin hasten da :

Justiziaren etsai odolgiroenak dirade bakea renak. Ezta a labadere zorihonik mündüan bakearen pare denik Haren begitha rtea ha in eder da nola ekhia, ha ren iza iria ren hatsa Arabiako intzens(u) ak beno maithagarriago. Gerlaren ezko süia denean hiltzen, suminak ehorzten, zelütik odeiak akhazatürik egün ederrak dereizkü eskentzen.

Bakea egoera hauskorra da :

Bakea galtho dianari ez (artzüka) üka emailearen eskütan hain eder da nola 
hartzalerenetan eta sekülan ez ederrago baizik zorihonak dereikünian, erre egiten delakoz, hanitzez bitoria benbo hobe delakoz Jinkoaren eskütan, berzea aldiz gizonarenetan.

Fortüna a izea bezala da ützültzen alde orotarat eztü egongia tiesorik, egün zonbaiten adiskide izanik, ezartzen da arrazo bagerik, gizon zaharra. Arren ez fida bakea eztela kotz ez eta kha rioegi haren eros lironea ri ezi joan ba da behin nekez da a tza maiten.

Gerlaren irudi itsusiak berriz gogoratzen zaizkio Egiategiri.

Gerletan garrak hiriak ta elizak dütü erratzen, lürak deseigoan ta galkiroan jarten, bere odolean bera ithorik, lürrak khapar ta elhorri baizik ez emaiten

Bakearen irudia berriz :

Jendea bakeari marrakaz hel dadin, bahiz sorhoen berderazitean elgeak erein berrian, jendea etxetarat deithzera, han aita, ama, haurrak miskandiak oro bakean, batzü berzeak ürgaisten dütieno, die Pa radüsiren üdürigoa. Bai bakea da Jinkoaren lloria ta lek"gdagoen herri güzieta $n$, han berdüdearen herria.

Egiategi apur bat urruntzen etxeko bakea aipatuz :

Bakeak dütü gauza güziak beren lekhütan jarten, legeak azkartzen, hoinak nekatzen; Bakea ezten etxean dago han infernüa. Eztaite(ke) bakerik bizi, algarri esküak eztieno emanik, faltsü ere bihotza hitzemanen berme ezpada. Bakeak dütü etxaltea $k$ xüxentzen, mendiak düen bezala ordokiak hontzen".

Gerla eta gatazka :

Gerlarik ikhusi eztianak, bakearen balioa eztaki. Filsofoek dereikie erraiten bakia eztela gauzetan (ekidran) orden ederra baizik, dena gogamenen (erakida) adostasun ta borhondadea gizonetan. Alabadere bethi bakean baratzea debeiagarri lizate(ke) Aharra arhin batek arrapizten dü batzütan adiskidegoa. Algarrekin bizi behardünetan ber gauza ta amorioa ezpadie bakeak lüzez iraün ezpeitate (..) O ! Etxaldean jendea eztenean ber gogotik nola la iteke parropian, herrian erresuman.

Anekdota, gaiztotik gaiztoagora :

Jaun bat bazen hiri batean jende güzien tirano; kharrikan zelarik igaraiten emazte zahar bat ba thü zian belhariko zereionak erran

- Jinko honak dereizüla Ja un maitea bizitze lüzea osa garrian.

(Huillantï) hurbildu zen emazteari ta galtho egin zertakoz zian Jinkoa harentako othoizten zakialarik parropianter in a ha la ari zela. Emazteak

- Ba i ja una hori zakit, zure a ita so Ja una ezagütü di(z)it a itoren sememetan ezpeitzen gizon bobenena, haren semea zen zure aita zena ga istoago zena, beldürrez zure jaun semea zü beno gaitzago gert'dakigün bizitze lüze bat dereizüt opatzen.

Gerlaren itxura berriz.

Gerlak dütü erresumak khanbiatzen, errepublikak desegiten, ererelijionea iharrausten, ha urra ri ünhüdia (ehaiten) hiltzen, a ita ri semea ehortz eraziten. Bakeak baizik desorden harrigarri hek aitzinta edo heltû diradenean medika eztiro. Jinkoaren manhüetan dago bakearen zori hona, heeetarik gibeltzen giradiano bakea da ürrhüntzen. 
Mandamenduen irakatsia :

Nahi die manamentuek (a ginduek, Ja inkoaren manda mentuek) etsa ia $k$ guha ur bezala deitzagün maitha ta ahalena dütügü eskerniatzen ta (kaltarraiten) ta gure elhetzaz edo obretzaz egünkal etsai berri dügü egiten. Bakea zen enea niano, haustü düdan bezain sarri berzerena dago ta heen borhondadea hartara ezpada, küpera eztirot. Gaitz handi baten gibeltü nahiz bakeaz niz gabetü, bena gogo ez emanez gaitzurre handiagoala, non doala, hartara niz erori. Jinkoak ta Izariak dereikie bakea gomendatzen, delakoz egiazko zori hona; bena hitz faltsüez ez eros (artzakian) baldintzan, ez ahatz hirur suerte direla :

1.a Hitz emaitean eztenean etxeki na hia, desleialtarzüna dena

2.a. Hitz emanaz garbaioa, a stokeria dena

3. a Hitz etxeki ezina, ganbero ta ohointsüen beltzakia dena

Egiategi, azkenean, gerla ezaguturik bakearen maitale da eta bakoitzak bere burua defenditzeko baizik eztakusa armada. Berak begi hobez ikusten du ofiziozko armada bat iraunkorra ez eta erregimentu kanbiakor horik erdi ofiziozko lagunkein antolatua.

\section{Egiategi eta errelijione jasangarritasuna}

Egiategi erreformiste dela diodanean erran nahi du bere hiru liburuen artean Eliza gizonen kritika zehatza badela eta Erromako poterearena ere. Ikus dezagun :

\section{Errelijioneaz}

Atal horretan kontzientzia askatasunari buruzko hitzaldi batekin hasten da :

Errelijionea da kontzientziaren ondarea, ihorek hartan züzenik eztiana, ezetare ta lazkida a hal lirokiana. Bortxaz edo a morioz hel a hal daite(ke) a nimal basaena, bena errelijione okherraren xüxentzea da Jinkoaren obra (...)

Karlos Kintoren errelisione gerlak aipatzen ditu eta nola katoliko printze batzuk Alemaniako protestanteen alde jalgi ziren. Gero gogorki kritikatzen du Luis XIV. Frantziakoa, protestantekin XVII. mendean erdi bakea zelarik gerratan sartu zelako eta protestanteak ihes egitera behartu :

Luis XIV zütialakoz errelijionean bere pekoak eskerniatü, gerla tristetan zen sarthü, milion ba tgizon Frantzia tik desterra tü ta deus honik obra tü etzela kotz Jinkoa ri a ga rada.

Kapituluaren azkenetan (360. o.) bere esperientzia kontatzen du :

Prusiako erresuman bada errelijione güzietarik ta heen artean ez aharra bakhoitzik (...) printzearen legeen indarretik gerthü bakeak düa la han egongia.

Ez ja arauki hainbeste nazio diferentetako herrian, ezi arrotz heetan Kalbinista frantsesek saldoa dienak handi ta erresuma haren izairitzu taldea nork eztü ezagützen; a labadere Alemanak ta Prusianak beza in mantso daude bakean, hirur Johane Lili bethi bihotzean dütükiela hilarteo dioelarik"

Jesuiten errua salatzen protestantekilako bakea (Edit de Nantes) nola hautsi zuen Luis XIV. renak (361.o.) :

Gerthaldi lotsagarri heen landan nola sar daite(ke) gizonaren bürü hünean Lois XIV bere konfesoreaz enganatürik Nanteseko Edita errebokatzen atrebitü zen ta Jesuiten 
a morioarengatik bere photerea galtzera, herena; zilegi etzaikü Jinkoaren(akadoietan) epaietan so egitea bena zirelakoz Jesuitak lür güziaz hügüntürik dirade orai Judioak bezala, lür güzian herraturik

Ondoko esaldiak oso trinko eta argi dira beste errelijioneak errespetatu behar direla diolarik :

Eztakit a rrazoan daudenez hotxki bizi behar giradea la gure errelijionean eztirenekin, ezi güziak girade Adamen seme ta aurrhide, errelijionen diferentziek askazigoa desegin eztiro. Uste ba düt nere errelijionea anglesa rena beno hobe dela, ha ren gogoan khinperra dago. Enean ezta legerik harena dezadan guda, berean da hain gizon pherestü nola ni enean, ni enean beno anglesa berean lea lago.

Errelijionen ugaritasuna Jainkoak nahi du :

Jinkoak eztüanez nahi erresuma güziek nork bere gisala lezen zerbütza, beren legen arauela, nork daki? Ta sinhestean amorioan ta garbaian dagoela errelijionearen igabürra nork eztaki.

Kristau Eliza gizonek eta oraingo ulemek duten proselitismoa ez luke onartuko. Zergatik bestei, ekonomia, politika presioen bidez beren sinesteak kendu eta gureak sartu?

Jenderik ezta aiten errelijionea etzereienik hobenena, ha rta koz berri ba ten aipa menak ohiltzen eztiana. Gauza zaharretan dügü fedea hain tinko non sinhets ezpeikiniro gure aitasoak ibili direla bide okherrean : hartako Indianoak zioan nahiago zela bere aitekilan infernian, eziez khiristien la gün pha radüsüaren esperantzan

Egiategik jarraitzen du :

Errelijionez egiazkoak berzer eztü mesperetxürik ekharten. Eta a dibideak ematen ditu: David eta Hiram paganoa Salomon eta Seleucos paganoa. Bibliatik beste bospasei adibide. Gregorio XIII eta frisiar paganoak, erromatar kristau eta Bandaloen arteko harremanak goresten

Hona testu luzean perpaus jakingarria :

Nor berean dagoeno berzeak berean beza ütz bizitzera faltsü heen iretziak izanik ere (..)

Erlisio gerretaz dio :

Errelijionea ren a stakürüan gerla, ha ri a rrotz ziradea nak ta hkapa ha renpean zonbat latzeria ta herio mündüan. Zonbat eliza errerik ta zenbat popülü eskeletürik. Ezta errelijionerik gerla manhatzen düanik: güziek bakea düe pheredikatzen, phüntü hartan diradea la rik bizitzea, zeren errelijionea heen a harren a razorik eztauke.

(...) Printzek ere zonbait aldiz beren onkarriak borroskan hetan nahasi zütielakoz, gerla errelijionekoak dirade sorthü beren artean, nahiz nork bere sekta edo fabori züana balia erazi; bena beren abantaillentzat baizik eztiradiano ari popülüak ere sinhestian errelijionearen ga tik ziradea la güdatzen, hartakotz gerla suerte ha u da bethi gaitzena izanik, ühaitz odolak ixurerazi, erresumak eskeletü ta errelijioneak irapaizi beno haboro galdü "

Egiategik ez ditu sinesleak gogorki kritikatzen sekta sortzaileak baizik teologoen arteko gatazkak sorturik, baina dio onartu behar direla beren sektariak zintzoak badira. Egiategik sekta hitza darabila katolikoentzat ere, eta hasierako erran nahiaz, alegia aurrekoekin moztu duena. Hain zuzen Judioen dekadentzia judaismoaren barneko gatazkek piztua izan dela dio. 
Erlisio arazoekin jarraikiz jadanik Luis XIV. en aitzi egin salapena agertzen zaiku (360. o.)

Khiristia khiristiari, frantzesa frantzesa ri lothürik, a la noa a la noa ri bezala zer da hantik heltü, erresumako elitea herri arrotzetara (jestegitürik) iheseginik, erresuma herenaz zelarik flakatürik ta herenaz haren etsaiak indartürik.

Eliza gizonei bizkarreratzen die errelijione nahaskeriak :

Aphezek alde batetik, ministreek bestetik a rotz (bohaderare) ha uspoaren ofizioa zieno zinez egiten.

Urruntxeago esaldi luze batean argi bat :

Nor bera da bere kontzientziaren nausi ta Jinkoa berhera haren (akadoi) epaile bakoitza...

Dezan nork berak jarraik zelüak eman dereion bürübidea ezi hau da gizonaren eginbidean arau xüxenena ta halakoa da Apostoliren irakastaldia. Kannon'khaldiz pha radüsiko borthak eztirade phorrikatzen ezetare heetan jendea bortxaz sar eraziten.

Dezagün arren Jüdioa, Calvinista, Lütheriana ütz beren sinhestetan ka tholikak beza in khiristiak dirade bi azkenak; halako Jinkoak nahi ezpalütü hitz bakhotx nahi bezala lütüke. Jüdioak ta Türkak beren errelijionen gozomen osoan dütü üzten ta ez khiristiarer Israelitek beza in Kananeanen eretzean aritzez, ez ha la ko manürik heen kontra egin.

Egiategik errelijioneak dituzten akatsak gora eta behera beharrezkoak direla idazten du eta ezagutu duen Alemaniaren kasua ematen, baina ikuspegi materialista batez ekonomia ekarpenak azpimarratuz :

Errelijione gaberik erresuma lerden ezta oke, harek dü jendea biltzen ta bizi beharrerat lürrak lantzen. Alemaniak noizpait herri oihan ta landa baizik etziana, jende gutik ta basa zen Strabonen ta Cautaren errraneala, orai da Europako herri ederrena halakatü dia la koz errelijione khiristianak.

Jakingarria Kanaandar eta Israeldarren arteko guduka hura zeren gure mendean piztu eta jarraitzen baitu, ez besteen konbertitzeko haien lurrez jabetzeko.

\section{Paganoetzaz}

Lehenagoko paganoetzaz mintzatzean Egiategi hasten da giristino martirioekin.

Paganoek herriaz zütien Khiristian lehenak salatzen eta meskabü heltzen zereitzeetan heer ogena emaiten. Tybria Italian bazen gainthü, Egyptan Nilak ordokiak ezpazütian bere hurez estaltzen khiristian sakrilegioak ziradean iturriak. Orozbat gosetzeak ta hilkorrak ziradea nean hiltzen; hartan beren Jinko faltsier grazia othoizteko khiristianak lehoner, tigroer ta hartzer zütien barrükieta ra urthikitzen ta bizirik janeraziten (...)

Neron enberadoreak Erromari sü züanean eman, jakile hanitz, erran lezen khiristianek züela phiztü, pagatü ta heetarik izigarri erre erazi, berak ziolarik zinez errelijione khiristia heltü geroztik galkiro güziak erresumari erori ziradeala.

Egiategik kontatzen du nola paganoak aski izpiritu zabalekoak ziren Kristo beren jainkoen artean hartzeko eta ohoratzeko Alexandro Severok egin bezala. Pagano batzuen egitate zintzoa aipatzen du. 
Plina bigarrenari dügü eskerra haren denboran khiristianek üken züen bakeaz ta Trajan enberadorearen manüetzaz heen fabori.

(...) Tyberak ere berze alte Senatüan galtho egin züan J.C. jar lezen beren jinkoen herrokan.

(...) ta Sybilla Cumanaren bersoak Virgilan diradeanak hanitz pagano khiristiatü zütien. Egiategiren obran zernahi kapitulutan lehenagoko paganoen bertuteez mintzatzen zaiku bai eta musulmanak eta beste eredu bezala emaiten.

\section{Aphezetzaz}

Egiategik nahi luke apezak beren lekuan egon ditezen eta honela hasten da kapitulua apezek besteen egitekoetan sartzenegi direlako eta diruzaleak direlako, ardien irudi pollita erabiliz :

Aphezak nahi güntüke güziak Santü, bena berze jendeak bezala gizon dirade. Zor zaien errespetü osoaz gozatzen eztiradeanean berek die ogena, jendearekin direlakoz akortatzen, jakin minez ere berzeren egitekoetan sartzen barnegi ta (diapego) lotura soberanak düalakoz mespretzüa soreraziten.. handinaia, iritzia ta zekeingoa batzüer dereitze menpenean iraunarazten.

Artzangoan aldiz arresak (a rdi kenteak) ordü beno lehenago dütiela mozten ta da kiela ezin hobeki ilheaz baliatzen; bena bada heetan gizon santürik berentzat deusere begiratzen eztienik, hanitz ere gizon ezin pherestägorik a phezgoari ohore dereitzenik.

Apezen ez ezkontzea paganismoarekin lotzen du Egiategik :

Noizpait aphezak ezkontzen ziradean Hildebran Aita Santia da lehena aphez a lemaner ezkontzea debeka tü zereiena. Ethiopian a phez güziak emaztedün dirade. Eliza lehenean ere lege zaharren araueala züenek sakrifikatzen etzienek ere ziradean ezkontürik Denys Constantinopleko aphezküpia bere lagüner zian gomendatzen aphez ezar ez lezen kastidadearen lege gogorra.

Gero Nizako kontzilioaren debekua aipatu ondoren, baina :

Vienako kontzilioan erabaki zela aphezer phuntzelekin ezkontzea zilegi zatekeala, heltü zena 89. urthean Formosus Aita Santiaren baimenean.

Apezgoaren antolakuntzaz gogoratzen du lehen aphezek beren zaharren artean apezpikuak hautatzen zituztela. Elizak bere justizia bereizia zuela. Kontatzen du Justianoen denbora kriminalean eta zibilean enperadoreen peko zirela, bakarrik Karlos garbala (Karles bilhogabea) izan zela apezpikuei apezen gaineko justizia eman ziena. Pierre Besseren teoriak gogorki kritikatzen ditu, XVI. mendean apezak Birjina baino gorago eta Jainkoaren maila berdinean ezarri zituelako Egiategik dio Jesu Kristoren erranak jarraikiz, ez direla estaduaren egitekoak Elizakoarekin nahasi beha :

Jinkoak dio "Bihür bedi Cosari harena dena"

Lehentasuna ematen dio printzeari :

Denbora zaharrenetan photerea printzetan za goela da go proba Valius Enperadorea ren gobernuan frairen egongiak zütianean legarrrez kargatü ta heen koskariak ta gazteak guda rigei har litean. 
Irteera.

Mugatu naizela diot ezen euskal Herriaz diona bizpahirur ikerlanetan zabalkiago ikertu dudalako. Laburzki errepikatzeko bere denboran bizi moldeen hobetzeak, demografiaren goratzeak haren ustez diruaren mirespenak gizartea desegiten zuten.

Adibidez XVIII. mendeko euskal soszale ezkointza jorratzen du, amodioz egiteko partez diruarengatik egiten direlako, hala bi gatü zakü batean sartzen; doteak goregi zirela Doteak nahi dütie ezkontzetan handi eta hurtzen dirade elhürra bezala . eta horretarako jendea zorretan jartzen, eta eztei egunetan dotea erdi jaten, diruaren agertze berri horrek Egiategiren ustez herriaren, familiaren, auzoen arteko elkartasunaren desegilea da.

Hemen irudi luke liberalismoaren aurka dela, baina gaur liberalismoaren alde direnek ez dute berdin pentsatzen, familiaren desegitea, lanaren erdainua, aisialdiaren gorestea gaurko baloreak direlako.

Bestalde apezek herriko plazetan dantza egitea debekatzen edo kontrolatzen dutelako eta hori XX. menderaino, neska mutilek, XVIII.mendean ostatuetan edanaz, mozkortuz, besta egiten zuten.

Euskal Herria utzirik, orduko Europara hedatzen ditu kritikak, batik bat Frantziara. Eliza jorratzen du ez bakarrik bere sineste jasangaiztasunaren gatik, baina proselitismoz beste erlisioneak galarazi nahi dituelako ; txarrago dena Frantzia bezalako herri giriristinoan eliza gizonek ebanjelioa utzirik politikan sartuak dabiltza.

Halaber kapitulu anitzetan orduko eta aintzinagoko erregeak begitan ditu ; alde batetik nobleak Kortean bilduz beste eginkizun eta ekimenetarako antzutzen baititu. Bai Luis XIV rekin hasirik Kortea Erregeren antzoki bilakatu zen, eta nobleek iraun edo igoain nahi zirenean erregeren lakaio neskato edo ohaide bilatzen ziren. Die egiten du euskaldun noblei Euskal Herrian egon ditezen. Hain zuzen bere liburua aitonen seme bati zuzendua da : lehen hamabi kapitulu guziak noblei die bat eginez urrentzen dira. Belako familia luzaz eredutzat ematen da, ezpaita Kortera joan baina herrian bertan lan egin. Jean Philippe de Bela generala haren nausia zitekeen.

Korteeko gastuek eta erregen gerlek herria mixeriara bultzatzen dutela bizkarretazen die, eta hori protestanteen erbestea salatu duen bezala. 